\title{
Effects of Ultrasound Irradiation on the Preparation of Ethyl Cellulose Nanocapsules Containing Spirooxazine Dye
}

\author{
Julija Volmajer Valh,, Simona Vajnhandl, ${ }^{1,2}$ Lidija Škodič, ${ }^{1}$ \\ Aleksandra Lobnik, ${ }^{1,2}$ Matejka Turel, ${ }^{2}$ and Bojana Vončina ${ }^{1}$ \\ ${ }^{1}$ Faculty of Mechanical Engineering, Institute for Engineering Materials and Design, Laboratory for Chemistry and \\ Environmental Protection, University of Maribor, Smetanova 17, SI-2000 Maribor, Slovenia \\ ${ }^{2}$ Institute for Environmental Protection and Sensors, Beloruska 7, SI-2000 Maribor, Slovenia \\ Correspondence should be addressed to Bojana Vončina; bojana.voncina@um.si
}

Received 8 March 2017; Revised 8 May 2017; Accepted 25 May 2017; Published 6 July 2017

Academic Editor: P. Davide Cozzoli

Copyright (C) 2017 Julija Volmajer Valh et al. This is an open access article distributed under the Creative Commons Attribution License, which permits unrestricted use, distribution, and reproduction in any medium, provided the original work is properly cited.

This article presents the influence of low frequency, high intensity ultrasonic irradiation on the characteristics (average size, polydispersity index) of ethyl cellulose nanocapsules encapsulating a photochromic dye. Photochromic nanocapsules were prepared by the emulsion-solvent evaporation method. The acoustic densities entering the system were systematically studied with respect to their abilities to modify and reduce the average sizes and polydispersity indexes of the nanocapsules. Scanning electron microscope, confocal laser microscope, and dynamic light scattering were utilised to characterise the structure, shape, size, and polydispersity of ethyl cellulose photochromic nanocapsules. We were able to tailor the size of the photochromic nanocapsules simply by varying the acoustic densities entering the system. At an acoustic density of $1.5 \mathrm{~W} / \mathrm{mL}$ and $60 \mathrm{~s}$ of continuous irradiation, we were able to prepare an almost monodispersed population of the nanocapsules with an average size of $193 \mathrm{~nm}$.

\section{Introduction}

Encapsulation is a very important method for improving the stabilities and protection capabilities of labile substances against degradation factors [1]. So, encapsulation of photochromic dyes is an effective method for protecting dye against environmental factors such as oxygen, $\mathrm{pH}$ value, and light, which lead to oxidation and deterioration of the photochromic dyes $[2,3]$. Photochromic dyes can be covalently bound to backbones [4] or immobilised by doping in polymer solids [5].

Mass coloration and coating are by far the more common industrial methods of application of photochromic colorants. Conventional methods to apply photochromic substance on fabrics include exhaustion dyeing [6]. However, this method fails to provide sufficient space for photochromic molecules to accomplish the structural transformation, and consequently the photochromic effect is insignificant. In the case that photochromic dyes cannot be used directly for coloration as they do not have any affinity for the substrates the encapsulation is required [7].

Preparations of photochromic micro- and nanocapsules have been published by several authors [3, 8, 9]. Zhou et al. [3] prepared melamine-formaldehyde microcapsules encapsulating photochromic compounds (two spirooxazine dyes and two naphtopyran dyes) with particle sizes of less than $5 \mu \mathrm{m}$ by in situ polymerisation. Han et al. [10] described the preparation of styrene photochromic nanocapsules containing diarylethenes and spirobenzopyran. In order to obtain nanosized capsules with average diameters of $50-150 \mathrm{~nm}$, they adopted the miniemulsion polymerisation method. Photoresponsive devices require that either the nanostructure or the molecular structure be responsive to an external stimulus, in this case, light [11].

Spirooxazine dyes open their oxazine rings upon UV irradiation; the concentration of spirooxazine in its ringopened form increases. The ring-opened merocyanine structure has a larger conjugated molecular system and is one of 


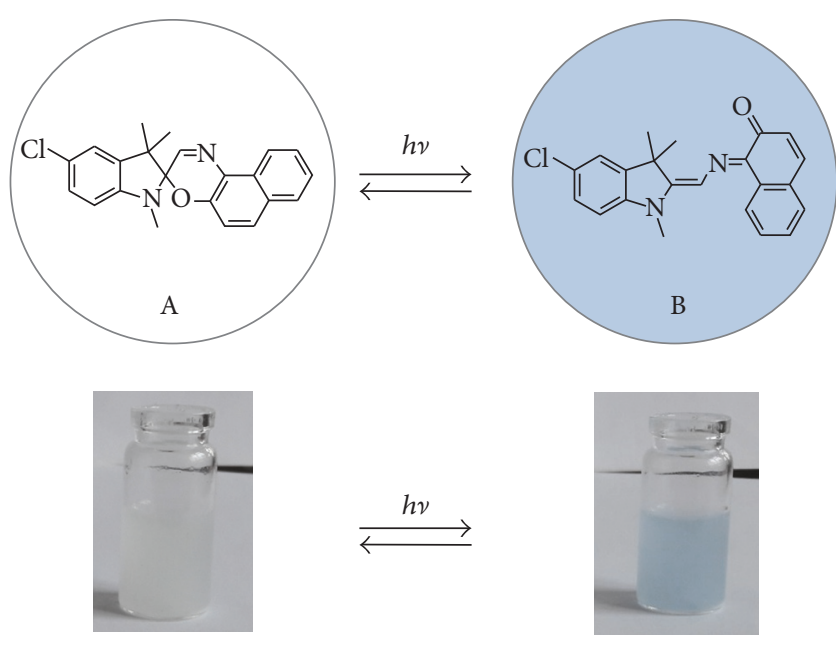

FIGURE 1: Schematic illustration of the photochromic effect of spirooxazine dye within a nanocapsule from "spiro" to "ring-open" form upon UV irradiation.

several photoisomers formed upon ring-opening. After UV exposure, the "ring-open" form returns automatically to a "spiro" noncoloured structure because the "spiro" form is thermodynamically a stable one [12]. A schematic illustration of the photochromic effect supported by photography of spirooxazine dye within a nanocapsule is shown in Figure 1. The ring-opened form has partial zwitterionic character.

Various methods have been designed to fabricate nanocapsules or microcapsules with various functionalities, such as chemical crosslinking [13], emulsion-solvent evaporation method [14], microfluidic fabrication technique [15], layer-by-layer self-assembling [16, 17], and as a novel encapsulation technology supercritical fluid extraction of emulsions (SFEE) $[18,19]$. Layer-by-layer method, as well as the SFEE method, can create nanocapsules with controlled sizes, composition, porosity, stability, surface functionality, and colloidal stability that can be applied as carriers for bioactive compounds [19-23].

In general the fabrication of nanocapsules is based either on the miniemulsion principle $[24,25]$ using the differences of interfacial tension, or on the phase separation process [26]. Amongst all the available methods, the polymer emulsionsolvent evaporation method is the more common [27]. The main issue is obtaining homogeneous dispersions. Mechanical stirring is often applied for improving the homogeneity of the dispersion but can hardly prevent particles from aggregation or agglomeration. In order to overcome this problem ultrasound irradiation has proved to be very efficient for eliminating agglomeration $[28,29]$.

Ultrasound irradiation of liquids induces a phenomenon called acoustic cavitation, which means the formation, growth, and implosion of microbubbles. The extreme temperature and pressure released during adiabatic bubble collapse are normally accompanied by shear forces and high speed microjects [30,31]. The powerful stirring effect of ultrasound is mainly due to the microstreaming caused by the oscillations of numerous resonant bubbles before they collapse. In this respect, intensities and durations of the ultrasound irradiation are important factors affecting the process. In general, to improve any process assisted by ultrasonic irradiation there exist a threshold limit and optimum operational parameters in the light of acoustic intensity, acoustic density, irradiation time, and so on needing to be determined.

This article presents a tailoring of the sizes of spirooxazine nanocapsules assisted by low frequency $(20 \mathrm{kHz})$ ultrasound irradiation varying the acoustic densities. An ultrasound probe system was applied with two different accessories, namely, replaceable and microtip, that differentiate between probe areas. The acoustic density entering the system and the influence of the latter on spirooxazine nanocapsules' shape, average size, and polydispersity index (PDI) were systematically studied. The influence of ultrasound irradiation time on nanocapsules formation was studied as well.

\section{Materials and Methods}

2.1. Materials. Dichloromethane, polyvinyl alcohol (PVA) $(\mathrm{Mw}=30.000-70.000,87-90 \%$ hydrolysed $)$, ethyl cellulose powder (EC) (viscosity $4 \mathrm{mPas}$, measured as a $5 \mathrm{wt} \%$ in $80: 20$ (vol) toluene/ethanol, at $25^{\circ} \mathrm{C}$ ), and 5-chloro-1,3dihydro-1,3,3-trimethylspiro[2H-indole-2,3-(3H)naphth[2,1b] $(1,4)$ oxazine] were obtained from Sigma Aldrich.

\subsection{Preparation of the Photochromic Nanocapsules}

Organic Phase. $70 \mathrm{mg}$ of ethyl cellulose powder was dissolved in $4 \mathrm{~mL}$ dichloromethane in $50 \mathrm{~mL}$ vessel using magnetic stirring at room temperature $\left(22^{\circ} \mathrm{C}\right)$. Different amounts of spirooxazine dye were added to the ethyl cellulose solution ( $5 \mathrm{mg}, 10 \mathrm{mg}, 20 \mathrm{mg}, 30 \mathrm{mg}$, and $40 \mathrm{mg}$ ). The final concentrations of the spirooxazine dye in solution were $1.25,2.5,5.0$, 7.7 , and $10 \mathrm{mg} / \mathrm{mL}$. Organic phase was continuously stirred at $600 \mathrm{rpm}$ (electric overhead stirrer IKA C-MAG HS 7) at room temperature $\left(22^{\circ} \mathrm{C}\right)$ until complete dissolution.

Water Phase. 1\% polyvinyl alcohol (PVA) water solution was prepared. The PVA powder was slowly added to water to avoid formation of lumps. Water phase was continuously stirred at $600 \mathrm{rpm}$ (electric overhead stirrer IKA C-MAG HS 7) at room temperature $\left(22^{\circ} \mathrm{C}\right)$ until the PVA is fully solubilized (at least $1 \mathrm{~h})$.

The size of nanocapsules, synthesised by oil-water emulsion process, can be affected by numerous parameters. One important step was to find a suitable emulsifier and adjust its concentration. In previous study [32] PVA was found to be a very effective surfactant in a similar system for emulsifying organic droplets in an aqueous phase [8]. According to previous study $1 \% \mathrm{w} / \mathrm{v}$ PVA was applied, and, upon ultrasound irradiation, nanocapsules were formulated. The increase of PVA concentration did not affect the size of the particles significantly [8]. Spirooxazine nanoparticles have been synthesised with $1 \% \mathrm{w} / \mathrm{v}$ PVA.

The photochromic nanocapsules were synthesised according to the method described in the previous work [8] with some modifications. Photochromic nanocapsules 
were prepared by an oil-in-water emulsion using the solvent evaporation method.

The oil-in-water emulsion was formulated when $10 \mathrm{~mL}$ of water phase was added drop wise into prepared organic phase in $50 \mathrm{~mL}$ vessel. The two phases were treated with mechanical stirring process (Procedure 1) or with ultrasound irradiation (Procedure 2).

Procedure 1. The two phases were stirred at a constant speed of $800 \mathrm{rpm}$ (electric overhead stirrer IKA C-MAG HS 7) at room temperature for 10 minutes in closed $50 \mathrm{~mL}$ vessels. The dispersion was then concentrated by continuous open-top stirring at room $\left(22^{\circ} \mathrm{C}\right)$ for 2 hours. Upon complete removal of the dichloromethane from the dispersion by the evaporation during mechanical mixing, the formed capsules were stabilized. To remove PVA, the capsules were centrifuged by an Eppendorf Centrifuge MiniSpin with 11,000 rpm for $30 \mathrm{~min}$ and supernatant was removed and redisposed within the distilled water for further analysis.

Procedure 2. The two phases were treated with ultrasound irradiation for different periods of time in continuous mode.

Different acoustic densities, determined via calorimetric measurements, and ultrasonic irradiation durations (30-180 s) were applied. Irradiation was performed using $20 \mathrm{kHz}$ low frequency ultrasound equipment from Sonics \& Materials. Two different ultrasound probe systems were used; probe with replaceable tip and with tip area of $1.3 \mathrm{~cm}^{2}$, and tapered microtip with tip area of $0.07 \mathrm{~cm}^{2}$.

All experiments were performed in an ice-water bath $\left(0-3^{\circ} \mathrm{C}\right)$ to keep the reaction mixture at constant temperature during ultrasound irradiation. The dispersion was then concentrated by continuous open-top stirring at room $\left(22^{\circ} \mathrm{C}\right)$ for 2 hours. Upon complete removal of the dichloromethane from the dispersion by the evaporation during mechanical mixing, the formed capsules were stabilized. To remove PVA, the capsules were centrifuged by an Eppendorf Centrifuge MiniSpin with $11,000 \mathrm{rpm}$ for $30 \mathrm{~min}$ and supernatant was removed and redispersed within the distilled water for further analysis.

All experiments were run in triplicate.

2.2.1. Characterisations of Ultrasound Systems. The transducer's efficiency for electrical to sound conversion was generally limited due to the fact that the real power dissipating into the liquid was determined by calorimetry. Acoustic power entering the system was defined using calorimetric measurements [33].

During the calorimetric measurements the cooling jacket contained air instead of water in order to minimize heat losses. For the selected amplitudes' measurements, the temperature $(T)$ was recorded against time $(t)$ at $10 \mathrm{~s}$ intervals using a thermocouple placed within the reactor. From the $T$ versus $t$ data, the temperature rise $(d T / d t)$ was estimated by fitting the data to a polynomial curve. The dissipated power was determined according to the equation [34]

$$
P_{\mathrm{diss}}=\left(\frac{d T}{d t}\right) m c_{p},
$$

where $c_{p}$ is the heat capacity of the water, $m$ is the mass of water, and $(d T / d t)$ represents the initial slope of the temperature rise versus time.

2.3. Scanning Electron Microscope. A scanning electron microscope (SEM) was used for observing the encapsulated spirooxazine dye within ethyl cellulose nanocapsules. The samples were examined using a Philips XL-30 environmental scanning electron microscope at $25 \mathrm{kV}$.

After synthesis the photochromic nanocapsules were centrifuged by an Eppendorf Centrifuge MiniSpin with $11,000 \mathrm{rpm}$ for $30 \mathrm{~min}$ and redispersed in distilled water for the scanning electron microscope measurements.

2.4. Measurements of Photochromic Nanocapsules Size and Polydispersity Index (PDI). Photochromic nanocapsules size and polydispersity index (PDI) were determined by the Zetasizer Nano ZS ${ }^{\circledR}$ (Malvern Instruments, Ltd., UK) equipped with DLS technology. Triplicate measurements were carried out using He-Ne laser at wavelength of $633 \mathrm{~nm}$ and at scattering angle of $173^{\circ}$ at $20^{\circ} \mathrm{C}$. Intensity weighted average hydrodynamic diameter was computed from intensity autocorrelation data using the cumulative method with DTS (nano) software (SOP) provided with the instrument. The $Z$-average diameter was reported as the mean droplet diameter of the emulsion. The PDI was a dimensionless measurement of the width of size distribution calculated from the cumulate analysis and ranged from 0 to 1.0. A small PDI value indicates an almost monodispersed population, whilst a large PDI indicates a very broad particle-size distribution. The calculations are defined in the ISO standard document $13321: 1996 \mathrm{E}$.

2.5. Confocal Laser Microscope. The confocal and multiphoton microscope system Leica TCS SP5 MP is suitable for deep imaging. The system has no limitation regarding IR laser configurations. Photochromic dyes in nanocapsules were detected using an argon laser at $488 \mathrm{~nm}$.

\section{Results and Discussion}

3.1. Effect of Ultrasonic Irradiation on the Characteristics of the Ethyl Cellulose Spirooxazine Nanocapsules. Preparation of ethyl cellulose nanocapsules encapsulating $10 \mathrm{mg}$ of spirooxazine dye was carried out using the oil-in-water emulsion evaporation process. After the addition of the water phase to the organic phase, the two phases were treated, firstly with the mechanical stirring process (Procedure 1), and, secondly, with ultrasonic irradiation in continuous mode for $60 \mathrm{~s}$ (Procedure 2). Ultrasonic irradiation was performed with low frequency probe type ultrasound $(20 \mathrm{kHz})$ using two different accessories, namely, the replaceable tip and the microtip. The systematic study of the influence of acoustic density on nanocapsules average size formations and PDI values was performed by applying different acoustic densities for the replaceable tip (Table 1) and for the microtip (Table 2), which differentiate in the active vibrating area.

Compared to mechanical stirring, low frequency high intensity ultrasound offers a better alternative technique during the preparation of more uniform sized capsules 


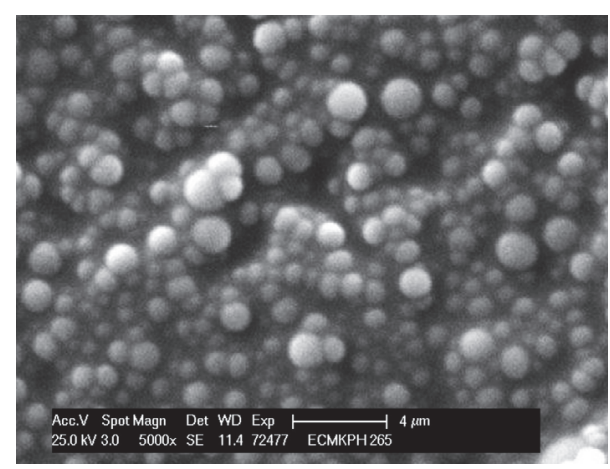

(a)

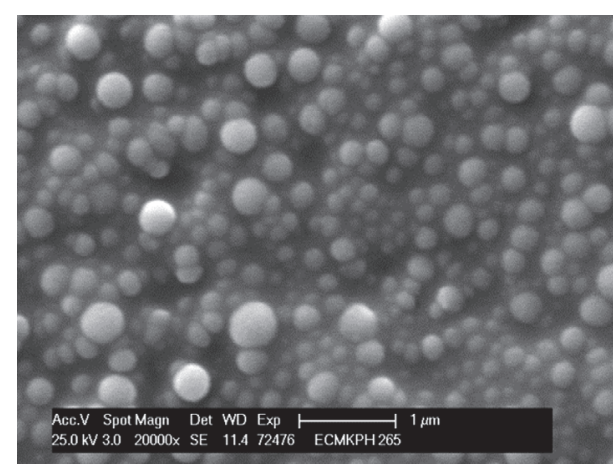

(b)

FIGURE 2: SEM images of ethyl cellulose nanocapsules containing spirooxazine prepared by mechanical stirring (a) and by ultrasound irradiation (b) (samples Nr. 1 and Nr. 3 from Table 1).

TABLE 1: Effects of acoustic density with replaceable tip on the average sizes and PDI values of ethyl cellulose nanocapsules containing spirooxazine dye.

\begin{tabular}{lccccc}
\hline $\begin{array}{l}\text { Sample } \\
\text { number* }\end{array}$ & $\begin{array}{c}\text { Amplitude } \\
(\%)\end{array}$ & $\begin{array}{c}\text { Acoustic } \\
\text { power } \\
(\mathrm{W})\end{array}$ & $\begin{array}{c}\text { Acoustic } \\
\text { density } \\
\left(\mathrm{WmL}^{-1}\right)\end{array}$ & $\begin{array}{c}\text { Average } \\
\text { size } \\
(\mathrm{nm})\end{array}$ & PDI \\
\hline 1 & 0 & - & - & 1304 & 0.728 \\
2 & 20 & 10 & 0.71 & 313 & 0.188 \\
3 & 30 & 21 & 1.50 & 193 & 0.067 \\
4 & 40 & 25 & 1.78 & 223 & 0.091 \\
5 & 50 & 36 & 2.57 & 231 & 0.077 \\
6 & 60 & 50 & 3.57 & 257 & 0.112 \\
7 & 70 & 67 & 4.78 & 279 & 0.128 \\
8 & 80 & 100 & 7.14 & 287 & 0.165 \\
9 & 90 & 115 & 8.20 & 404 & 0.193 \\
\hline$*$
\end{tabular}

*All experiments were run in triplicate.

TABLE 2: Effects of acoustic density using tapered microtip on the average sizes and PDI values of ethyl cellulose nanocapsules containing spirooxazine dye.

\begin{tabular}{lccccc}
\hline $\begin{array}{l}\text { Sample } \\
\text { number }\end{array}$ & $\begin{array}{c}\text { Amplitude } \\
(\%)\end{array}$ & $\begin{array}{c}\text { Acoustic } \\
\text { power } \\
(\mathrm{W})\end{array}$ & $\begin{array}{c}\text { Acoustic } \\
\text { density } \\
\left(\mathrm{W} \mathrm{ml}^{-1}\right)\end{array}$ & $\begin{array}{c}\text { Average } \\
\text { size } \\
(\mathrm{nm})\end{array}$ & PDI \\
\hline 1 & 0 & - & - & 1410 & 0.686 \\
2 & 20 & 6 & 0.43 & 276 & 0.127 \\
3 & 30 & 15 & 1.07 & 216 & 0.091 \\
4 & 40 & 22 & 1.57 & 227 & 0.118 \\
\hline
\end{tabular}

${ }^{*}$ All experiments were run in triplicate.

within the nanoscale range. Classical mechanical stirring was insufficient in the preparation of those photochromic capsules with average diameter sizes less than $1 \mu \mathrm{m}$ and with small values of PDI. Samples number 1 in Table 1 presented photochromic capsules prepared with classical mechanical stirring according to Procedure 1. The sizes of capsules synthesised without ultrasonic irradiation were above $1 \mu \mathrm{m}$ with PDI values of almost 1 , indicating a very broad distribution of the particle sizes. In contrast ultrasonic irradiation was essential for the production of uniform sized nanocapsules below $1 \mu \mathrm{m}$. Nevertheless, the results presented in Tables 1 and 2 indicated that the acoustic density had an important influence on the spirooxazine nanocapsules' average sizes and their PDI values regardless of the used concentration of dye. In the case of the replaceable tip a decrease in nanocapsules average size was observed with increasing acoustic density reaching the minimum average size of $193 \mathrm{~nm}$ and PDI value of 0.067 at acoustic density of $1.5 \mathrm{~W} / \mathrm{mL}$. After that the subsequent increase of acoustic density resulted repeatedly in gradual increases of the average sizes of those nanocapsules reaching the average size of $404 \mathrm{~nm}$ at maximum acoustic density of $8.2 \mathrm{~W} / \mathrm{mL}$.

This observation could be explained by too high vibrational amplitudes leading to decoupling between the vibrating plate and the liquid near the radiating surface resulting in a cloud of bubbles, which diminish the penetration of the sound into the liquid [35].

The tapered microtip had in comparison to the replaceable tip an almost 20 -fold smaller vibrating area. The same trend can be observed regarding the maximum plateau of acoustic density necessary for the minimum possible average size acquisition of nanocapsules with uniform size distribution as evident from the PDI measurements. In the case of the tapered microtip, an acoustic density of $1.07 \mathrm{~W} / \mathrm{mL}$ seems to be the optimum ultrasound parameter for the production of nanocapsules with average sizes of $216 \mathrm{~nm}$ and with PDI of 0.091 .

The particle sizes observed by SEM were in good agreement with the dynamic light scattering investigations. Figure 2 presents SEM images of ethyl cellulose nanocapsules containing spirooxazine dye prepared with the support of classical mechanical stirring (a) and with the use of ultrasound irradiation at acoustic density of $1.5 \mathrm{~W} / \mathrm{mL}$ and sonication time of $60 \mathrm{~s} \mathrm{(b).}$

We proved that with nanoencapsulation of spirooxazine dye the photochromic effect still remained. Photo-induced reversible structural transformation of incorporated spirooxazine dye still responded to the light. Upon UV irradiation the 


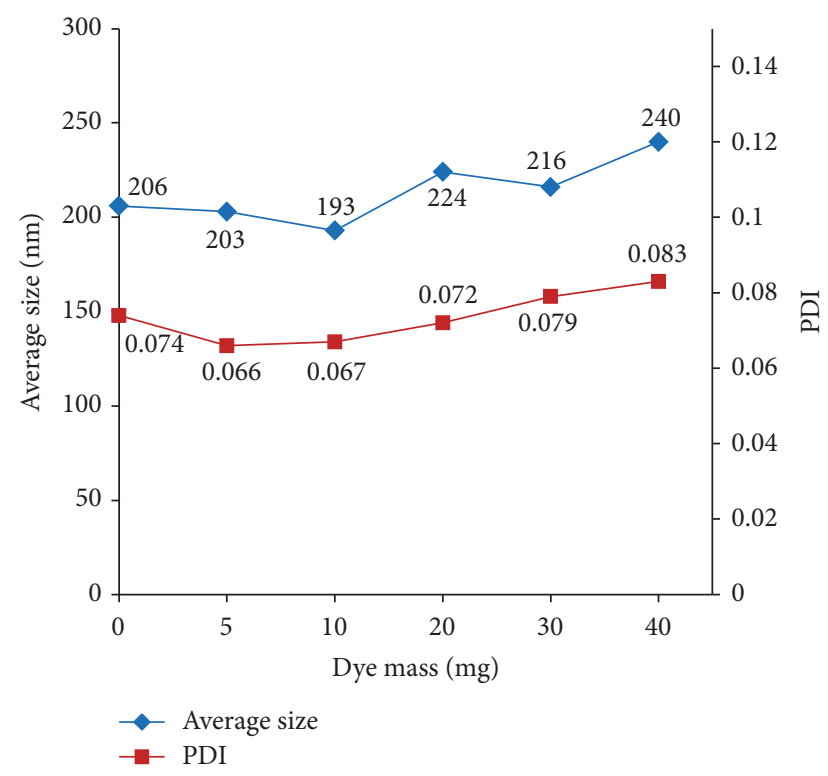

Figure 3: Average sizes (nm) and PDIs of spirooxazine nanocapsules in the presence of different spirooxazine concentrations.

color of the nanocapsules with spirooxazine dye turned blue (Figure 1) and then bleached within a few minutes. According to naked eye observation there were no significant differences in the photochromic effect when the sizes of the nanocapsules decreased to average size of about $193 \mathrm{~nm}$.

3.2. Effect of Spirooxazine Dye Mass. In this set of experiments, the effects of five different masses $(5 \mathrm{mg}, 10 \mathrm{mg}, 20 \mathrm{mg}$, $30 \mathrm{mg}$, and $40 \mathrm{mg}$ ) of spirooxazine dye in dichloromethane $(4 \mathrm{~mL})$ solution on the size distributions of photochromic nanoparticles were investigated. All experiments were performed with a replaceable tip at optimum acoustic density of $1.5 \mathrm{~W} / \mathrm{mL}$ and sonication time of $60 \mathrm{~s}$. The smallest, averagesized nanocapsules of $193 \mathrm{~nm}$ were obtained when $10 \mathrm{mg}$ of the dye was used. Increase in the spirooxazine dye mass from 10 to $40 \mathrm{mg}$ slightly increased the sizes of the nanocapsules, namely, up to $240 \mathrm{~nm}$ at $40 \mathrm{mg}$ of the dye. Nevertheless, low values of the polydispersity indexes in all cases indicated an almost monodispersed population of nanocapsules prepared under the above stated experimental conditions. We can conclude that the mass of the spirooxazine dye did not significantly affect the average size of the photochromic nanocapsules (Figure 3).

In parallel during the same experimental conditions nanocapsules in the absence of dye were prepared. The average size of the nanocapsules without the spirooxazine dyes was $206 \mathrm{~nm}$ with PDI 0.074 .

3.3. Effect of the Sonication Time. The influence of the ultrasonic irradiation duration on the spirooxazine nanocapsules average size was also studied. Experiments were performed with replaceable tip at $1.5 \mathrm{~W} / \mathrm{mL}$ of acoustic density with a spirooxazine dye mass of $30 \mathrm{mg}$, whilst we concluded that the masses of the spirooxazine dye did not significantly influence

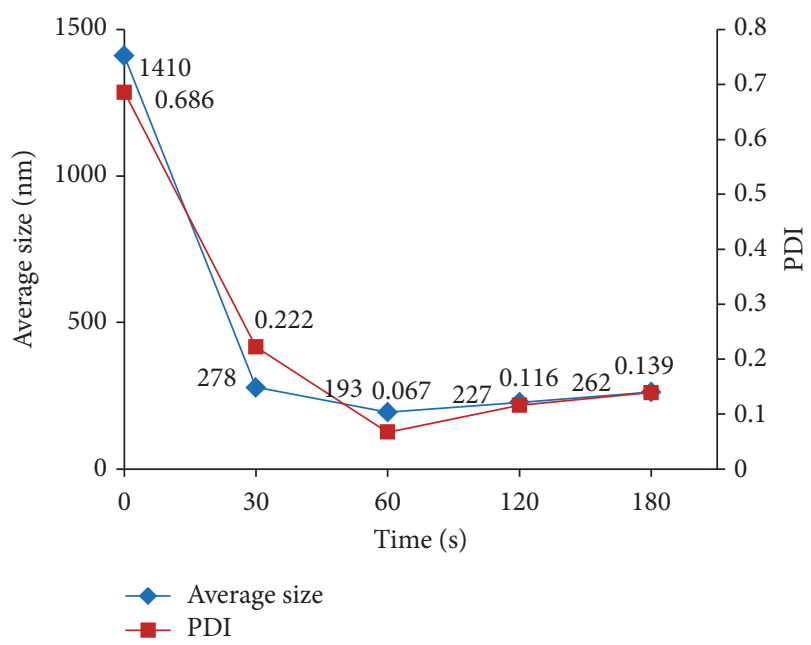

FIgure 4: Average sizes, PDIs of spirooxazine nanocapsules at different sonication times.

TABLE 3: Physical stabilities of the spirooxazine nanocapsules.

\begin{tabular}{lcccc}
\hline $\begin{array}{l}\text { Amplitude } \\
(\%)\end{array}$ & $\begin{array}{c}\text { Average size } \\
\text { (nm) }\end{array}$ & PDI & $\begin{array}{c}\text { Average size } \\
\text { after 6 } \\
\text { months } \\
\text { (nm) }\end{array}$ & $\begin{array}{c}\text { PDI } \\
\text { after 6 } \\
\text { months }\end{array}$ \\
\hline 20 & 270 & 0.178 & 242 & 0.174 \\
30 & 216 & 0.079 & 216 & 0.134 \\
\hline
\end{tabular}

Both experiments were run in triplicate.

the average size of the photochromic nanocapsules. Sonication was applied for 30,60,120, and $180 \mathrm{~s}$ after the addition of the water phase to the organic phase. From the results presented in Figure 4 it is evident that an optimum sonication time exists when producing nanocapsules of average size below $200 \mathrm{~nm}$. Any increase in irradiation times resulted in gradual increases in the average size. The reason for this phenomenon probably lies in the small working volume in which the ultrasound waves after a certain time period start to interfere and cause the so-called cushioning effect.

The nanocapsules diameter and polydispersity index sharply decreased as the ultrasonic irradiation time increased up to $30 \mathrm{~s}$. The smallest average size of nanocapsules was obtained at the sonication time of $60 \mathrm{~s}$. With prolonged sonication up to $180 \mathrm{~s}$ we were unable to produce nanocapsules below $200 \mathrm{~nm}$. Sample in Figure 4, at time $0 \mathrm{~s}$, presented photochromic capsules prepared via classical mechanical stirring (Procedure 1), rather than via ultrasonic irradiation.

3.4. Spirooxazine Nanocapsules Stabilities over Time. As mentioned earlier, spirooxazine nanocapsules were prepared by an oil-in-water emulsion using the solvent evaporation method, by adding the water phase to the organic phase. For physical stability measurements of $30 \mathrm{mg}$ of spirooxazine dye were used; the irradiation time was 60 seconds with acoustic densities of $0.71 \mathrm{~W} / \mathrm{mL}$ and $1.5 \mathrm{~W} / \mathrm{mL}$. Both experiments in Table 3 were run in triplicate. The spirooxazine nanocapsules prepared according to the Procedure 2 were stored in distilled 


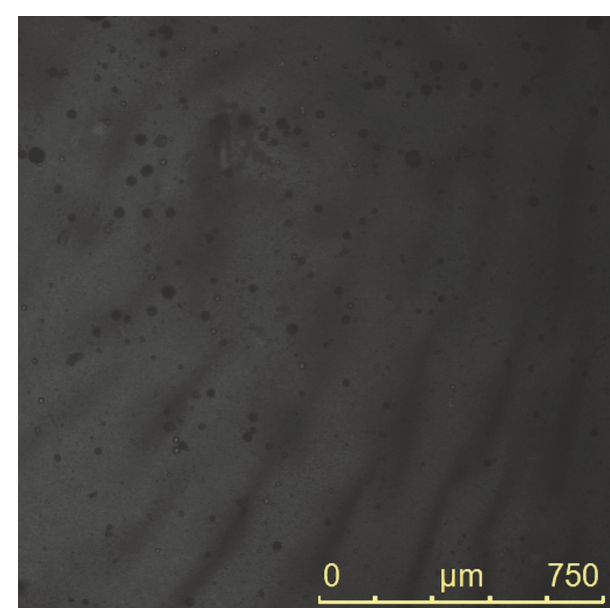

(a)

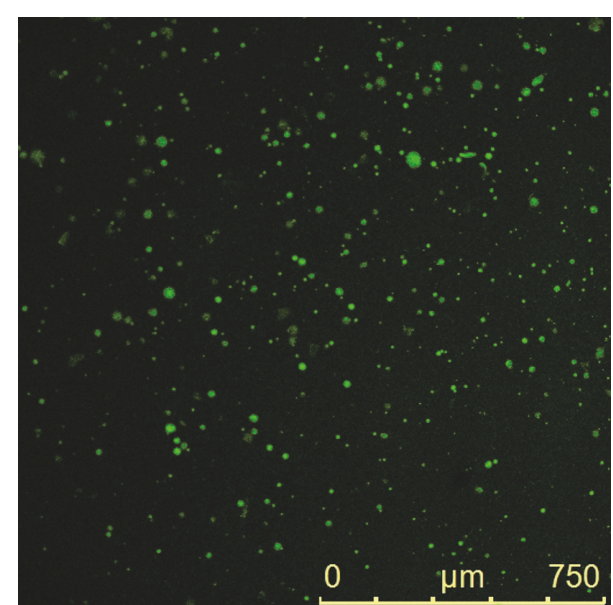

(b)

Figure 5: (a) Visualisation of spirooxazine nanocapsules by light microscopy image. (b) Confocal (CLSM) images of spirooxazine nanocapsules.

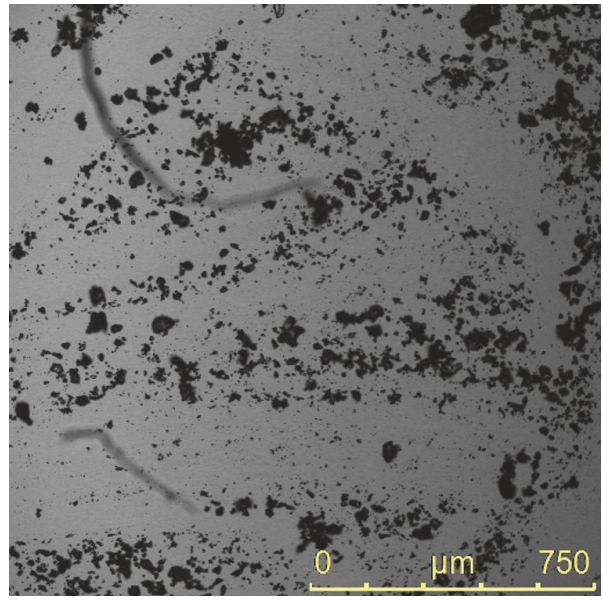

(a)

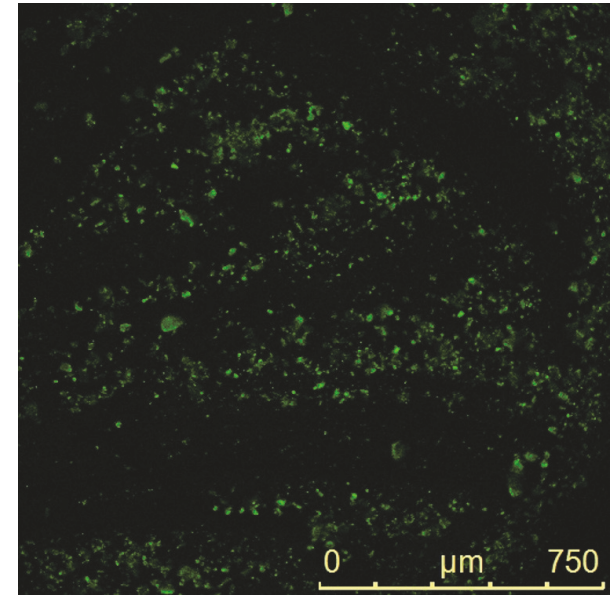

(b)

FIGURE 6: (a) Visualisation of spirooxazine dye by light microscopy image. (b) Confocal (CLSM) images of spirooxazine dye.

water at room temperature for 6 months. The results showed that the average size of spirooxazine nanocapsules did not change; moreover, under these storage conditions we did not observe any aggregation (Table 3). The photochromic effect during this period still remained.

3.5. Confocal Laser Microscopic Analysis. Spirooxazine nanocapsules, spirooxazine dye, and empty nanocapsules were characterised by the confocal laser scanning microscopy (CLSM), in order to confirm that the interior of nanocapsules is fulfilled with spirooxazine dye molecules. Comparably, the visualisation of spirooxazine nanocapsules was possible also using normal light microscope (Figures 5(a), 6(a), and 7(a)).

Spirooxazine nanocapsules, spirooxazine dye, and empty nanocapsules were excited with an argon laser beam at $488 \mathrm{~nm}$ and 10x magnification. CLSM images of spirooxazine nanocapsules are shown in Figure 5(b) and CLSM images of spirooxazine dye and empty nanocapsules are shown in Figures 6(b) and 7(b).

CLSM image (Figure 5(b)) of spirooxazine nanocapsules illustrated localization of spirooxazine dye within the ethyl cellulose nanocapsules. CLSM image of spirooxazine dye and CLSM image of empty nanocapsules confirm this.

As shown in Figure 5 the spirooxazine nanocapsules were spherical. Based on confocal laser microscope images we supposed that the significant part of nanocapsules interior corresponds to spirooxazine dye molecules.

\section{Conclusions}

The influence of ultrasound irradiation on the ethyl cellulose spirooxazine nanocapsules formation and average size distributions was systematically studied by varying the acoustic densities and process times. We were able to prepare 


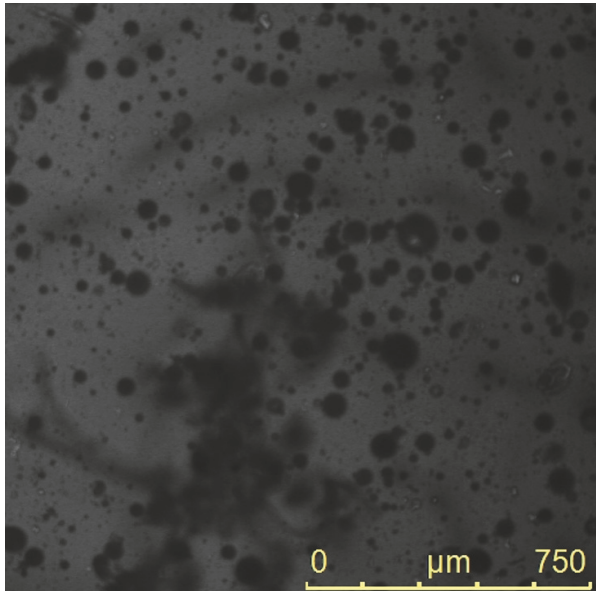

(a)

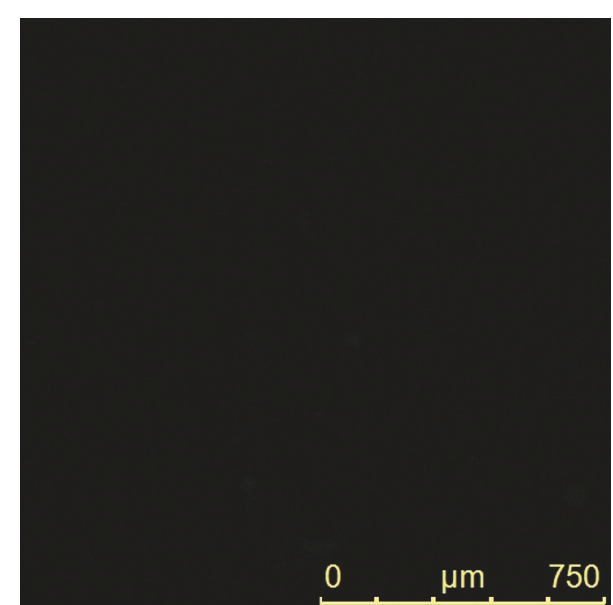

(b)

FIGURE 7: (a) Visualisation of empty nanocapsules by light microscopy image. (b) Confocal (CLSM) images of empty nanocapsules.

spherically uniform sized nanocapsules with diameter sizes below $200 \mathrm{~nm}$ simply by optimising experimental conditions related to the acoustic densities and irradiation times. At $1.5 \mathrm{~W} / \mathrm{mL}$ and sonication time of $60 \mathrm{~s}$ we obtained the smallest average size of spirooxazine nanocapsules, that is, $193 \mathrm{~nm}$ and PDI 0.067, prepared with $10 \mathrm{mg}$ of dye. In all cases the spirooxazine nanocapsules did not agglomerate and they conserved the photochromic effect over time.

Preparation of the uniform sized nanocapsules is important for future applications. Our future applications will focus on studies related to the photochromism as well as the incorporation of spirooxazine nanocapsules into silica matrices for sensing application.

\section{Conflicts of Interest}

The authors declare that there are no conflicts of interest regarding the publication of this paper.

\section{Acknowledgments}

The authors thank Dr. Tivadar Feczko from Faculty of Engineering, University of Pannonia, for SEM images, Dr. Irena Ban from Faculty of Chemistry and Chemical Engineering, University of Maribor, for providing facilities for measurements of photochromic nanocapsules' size and polydispersity index, and Mr. Rudi Mlakar from Faculty of Medicine, University of Maribor, for obtaining images through confocal laser scanning microscopy (CLSM).

\section{References}

[1] C. Preetz, A. Rübe, I. Reiche, G. Hause, and K. Mäder, "Preparation and characterization of biocompatible oil-loaded polyelectrolyte nanocapsules," Nanomedicine: Nanotechnology, Biology, and Medicine, vol. 4, no. 2, pp. 106-114, 2008.

[2] T. Cheng, T. Lin, R. Brady, and X. Wang, "Fast response photochromic textiles from hybrid silica surface coating," Fibers and Polymers, vol. 9, no. 3, pp. 301-306, 2008.
[3] Y. Zhou, Y. Yan, Y. Du, J. Chen, X. Hou, and J. Meng, "Preparation and application of melamine-formaldehyde photochromic microcapsules," Sensors and Actuators, B: Chemical, vol. 188, pp. 502-512, 2013.

[4] S. Wang, C. Yu, M.-S. Choi, and S.-H. Kim, "Synthesis and switching properties of photochromic carbazole-spironaphthoxazine copolymer," Journal of Photochemistry and Photobiology A: Chemistry, vol. 192, no. 1, pp. 17-22, 2007.

[5] I.-J. Lee, "A low temperature spectrophotometric study of the photomerocyanine form of spirooxazine doped in polystyrene film," Journal of Photochemistry and Photobiology A: Chemistry, vol. 124, no. 3, pp. 141-146, 1999.

[6] M. Parhizkar, Y. Zhao, X. Wang, and T. Lin, "Photostability and durability properties of photochromic organosilica coating on fabric," Journal of Engineered Fibers and Fabrics, vol. 9, no. 3, pp. 65-73, 2014.

[7] M. A. Chowdhury, M. Joshi, and B. S. Butola, "Photochromic and thermochromic colorants in textile applications," Journal of Engineered Fibers and Fabrics, vol. 9, no. 1, pp. 107-123, 2014.

[8] T. Feczkó, O. Varga, M. Kovács, T. Vidóczy, and B. Voncina, "Pr eparation and characterization of photochromic poly(methyl methacrylate) and ethyl cellulose nanocapsules containing a spirooxazine dye," Journal of Photochemistry \& Photobiology A: Chemistry, vol. 222, no. 1, pp. 293-298, 2011.

[9] T. Feczkó, M. Kovács, and B. Voncina, "Improvement of fatigue resistance of spirooxazine in ethyl cellulose and poly(methyl methacrylate) nanoparticles using a hindered amine light stabilizer," Journal of Photochemistry and Photobiology A: Chemistry, vol. 247, pp. 1-7, 2012.

[10] M. Han, E. Lee, and E. Kim, "Preparation and optical properties of polystyrene nanocapsules containing photochromophores," Optical Materials, vol. 21, no. 1-3, pp. 579-583, 2003.

[11] M.-Q. Zhu, L. Zhu, J. J. Han, W. Wu, J. K. Hurst, and A. D. Q. Li, "Spiropyran-based photochromic polymer nanoparticles with optically switchable luminescence," Journal of the American Chemical Society, vol. 128, no. 13, pp. 4303-4309, 2006.

[12] H. Dürr and H. Bouas-Laurent, Photochromism: Molecules and Systems, Elsevier, Amsterdam, Netherlands, 2003.

[13] K. H. Bae, Y. Lee, and T. G. Park, "Oil-encapsulating PEO-PPO$\mathrm{PEO} / \mathrm{PEG}$ shell cross-linked nanocapsules for target-specific 
delivery of paclitaxel," Biomacromolecules, vol. 8, no. 2, pp. 650656, 2007.

[14] Y. Zhang, H. F. Chan, and K. W. Leong, "Advanced materials and processing for drug delivery: the past and the future," Advanced Drug Delivery Reviews, vol. 65, no. 1, pp. 104-120, 2013.

[15] T. Watanabe, Y. Kimura, and T. Ono, "Microfluidic fabrication of monodisperse polylactide microcapsules with tunable structures through rapid precipitation," Langmuir, vol. 29, no. 46, pp. 14082-14088, 2013.

[16] I. S. Elizarova and P. F. Luckham, "Fabrication of polyelectrolyte multilayered nano-capsules using a continuous layer-by-layer approach," Journal of Colloid and Interface Science, vol. 470, pp. 92-99, 2016.

[17] J. Cui, M. P. Van Koeverden, M. Müllner, K. Kempe, and F. Caruso, "Emerging methods for the fabrication of polymer capsules," Advances in Colloid and Interface Science, vol. 207, no. 1, pp. 14-31, 2014.

[18] S. Zhong, H. Zhang, Y. Liu et al., "Folic acid functionalized reduction-responsive magnetic chitosan nanocapsules for targeted delivery and triggered release of drugs," Carbohydrate Polymers, vol. 168, pp. 282-289, 2017.

[19] C. Prieto, L. Calvo, and C. M. M. Duarte, "Continuous supercritical fluid extraction of emulsions to produce nanocapsules of vitamin E in polycaprolactone," The Journal of Supercritical Fluids, vol. 124, pp. 72-79, 2017.

[20] L. L. Del Mercato, M. M. Ferraro, F. Baldassarre et al., "Biological applications of LbL multilayer capsules: from drug delivery to sensing," Advances in Colloid and Interface Science, vol. 207, no. 1, pp. 139-154, 2014.

[21] A. C. Pinheiro, A. I. Bourbon, M. A. Cerqueira et al., "Chitosan/ fucoidan multilayer nanocapsules as a vehicle for controlled release of bioactive compounds," Carbohydrate Polymers, vol. 115, pp. 1-9, 2015.

[22] T. G. Shutava, P. P. Pattekari, K. A. Arapov, V. P. Torchilin, and Y. M. Lvov, "Architectural layer-by-layer assembly of drug nanocapsules with PEGylated polyelectrolytes," Soft Matter, vol. 8, no. 36, pp. 9418-9427, 2012.

[23] M. T. M. S. Gomes, D. T. Santos, and M. A. A. Meireles, “Trends in particle formation of bioactive compounds using supercritical fluids and nanoemulsions," Food and Public Health, vol. 2, no. 5, pp. 142-152, 2012.

[24] J. Su, J. Chen, F. Zeng, Q. Chen, S. Wu, and Z. Tong, "Synthesis and photochromic property of nanoparticles with spiropyran moieties via one-step miniemulsion polymerization," Polymer Bulletin, vol. 61, no. 4, pp. 425-434, 2008.

[25] F. Tiarks, K. Landfester, and M. Antonietti, "Preparation of polymeric nanocapsules by miniemulsion polymerization," Langmuir, vol. 17, no. 3, pp. 908-918, 2001.

[26] L. Zheng, Z. Ding, M. Zhang, and J. Sun, "Microencapsulation of bayberry polyphenols by ethyl cellulose: preparation and characterization," Journal of Food Engineering, vol. 104, no. 1, pp. 89-95, 2011.

[27] O. Grari, L. Dhouibi, F. Lallemand, C. C. Buron, A. Et Taouil, and J. Y. Hihn, "Effects of high frequency ultrasound irradiation on incorporation of $\mathrm{SiO} 2$ particles within polypyrrole films," Ultrasonics Sonochemistry, vol. 22, pp. 220-226, 2015.

[28] C. Sauter, M. A. Emin, H. P. Schuchmann, and S. Tavman, "Influence of hydrostatic pressure and sound amplitude on the ultrasound induced dispersion and de-agglomeration of nanoparticles," Ultrasonics Sonochemistry, vol. 15, no. 4, pp. 517$523,2008$.
[29] S. Abbas, E. Karangwa, M. Bashari et al., "Fabrication of polymeric nanocapsules from curcumin-loaded nanoemulsion templates by self-assembly," Ultrasonics Sonochemistry, vol. 23, pp. 81-92, 2015.

[30] K. S. Suslick, S.-B. Choe, A. A. Cichowlas, and M. W. Grinstaff, "Sonochemical synthesis of amorphous iron," Nature, vol. 353, no. 6343, pp. 414-416, 1991.

[31] K. S. Suslick, Ultrasound, It's Chemical, Physical and Biological Effects, VCH, New York, NY, USA, 1988.

[32] T. Feczkó, V. Kokol, and B. Voncina, "Preparation and characterization of ethylcellulose-based microcapsules for sustaining release of a model fragrance," Macromolecular Research, vol. 18, no. 7, pp. 636-640, 2010.

[33] S. Vajnhandl and A. M. Le Marechal, "Case study of the sonochemical decolouration of textile azo dye Reactive Black 5," Journal of Hazardous Materials, vol. 141, no. 1, pp. 329-335, 2007.

[34] T. J. Mason, Sonochemistry, Oxford University Press, New York, NY, USA, 1999.

[35] K. M. Swamy and K. L. Narayana, Ultrasound in Environmental Protection, JAI Press Inc., 2001. 

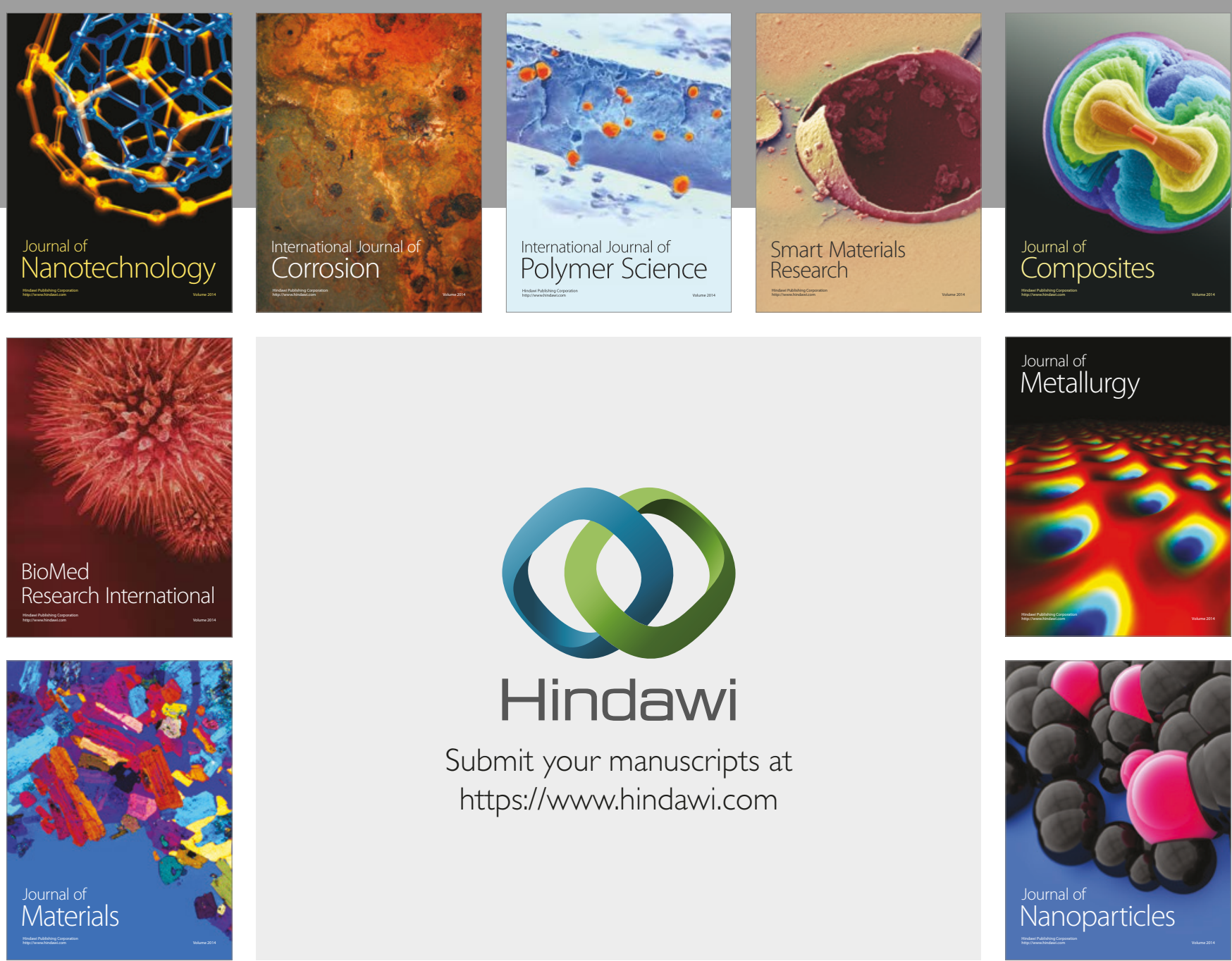

\section{Hindawi}

Submit your manuscripts at

https://www.hindawi.com
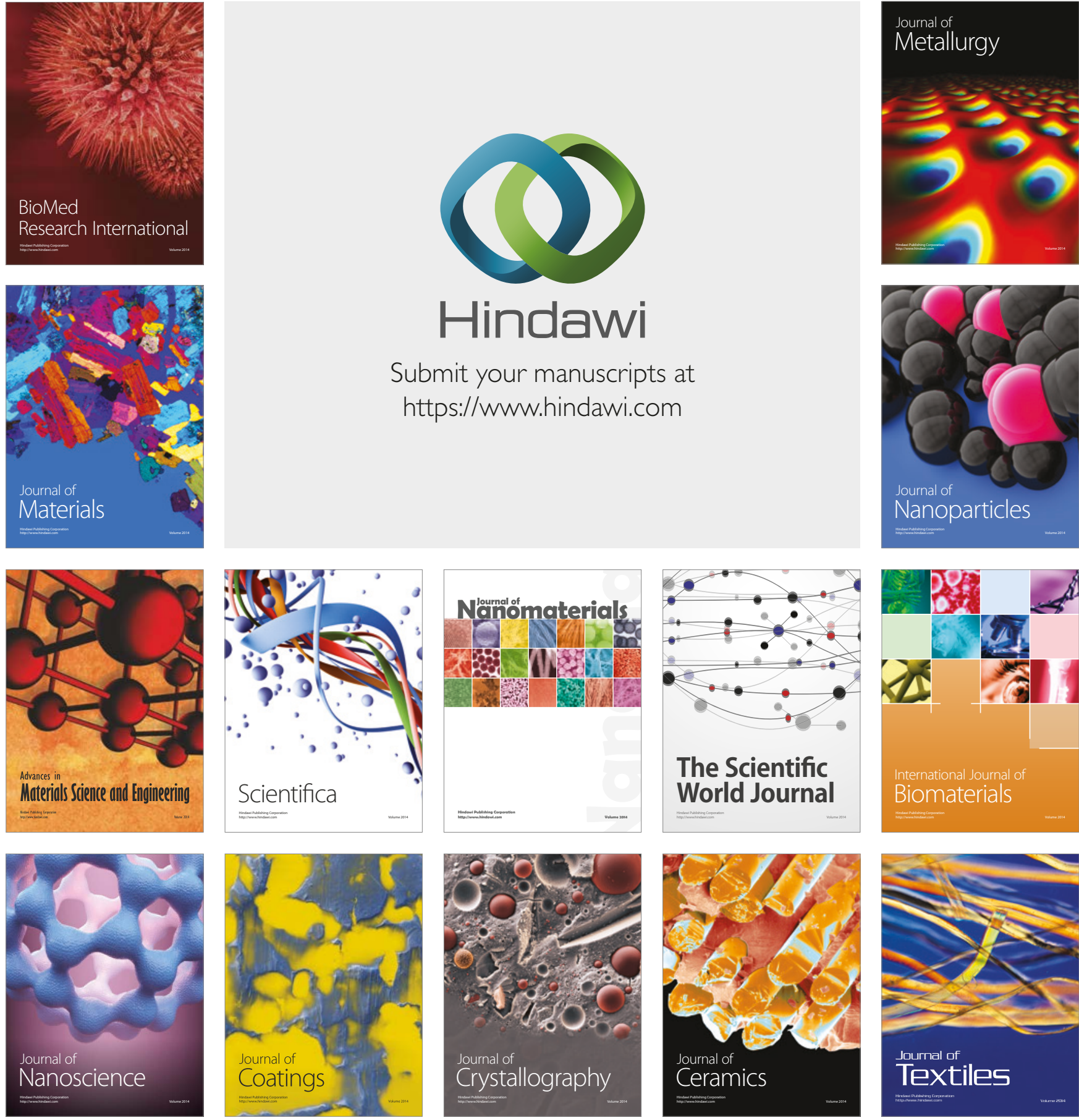

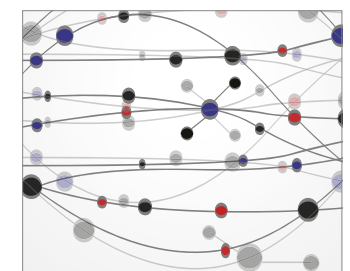

The Scientific World Journal
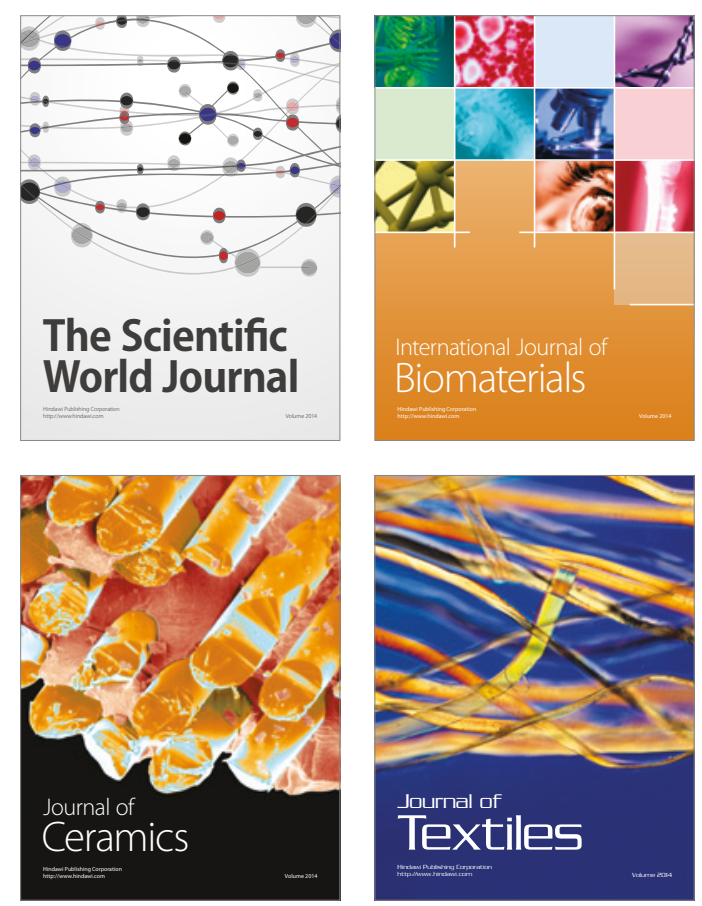